\title{
SATISFACCIÓN DE USUARIOS EN HOSPITALES PÚBLICOS: EXPERIENCIA DEL PLAN «CERO COLAS» EN ICA, PERÚ
}

\author{
Bladimir Becerra-Canales ${ }^{1,2, a}$, Ángela Condori-Becerra ${ }^{3, b}$
}

\begin{abstract}
RESUMEN
Con el objetivo de conocer el nivel de satisfacción de los usuarios de consultorios externos de cuatro hospitales públicos de la región Ica luego de la implementación del plan «Cero colas» se encuestó a 1067 usuarios, utilizando un instrumento que indagaba sobre las características generales, calificación del servicio y satisfacción global. Se encontró un nivel de satisfacción global «muy satisfecho/satisfecho» del 72,5\% en el Hospital de Apoyo de Nazca, 70,6\% en el Hospital Santa María del Socorro de Ica, $72,1 \%$ en el Hospital Regional de Ica y 73,7\% en el Hospital San Juan de Dios de Pisco. Se concluye que la implementación del plan «Cero Colas» mejoró el nivel de satisfacción global, el buen trato, el cumplimiento del horario de atención médica y la claridad e información brindados por encima del estándar esperado.
\end{abstract}

Palabras clave: Satisfacción del Paciente; Calidad de la Atención de Salud; Atención Ambulatoria; Encuestas de Salud; Perú (Fuente: DeCS BIREME).

\section{USER SATISFACTION IN PUBLIC HOSPITALS: EXPERIENCE OF THE «CERO COLAS» («ZERO QUEUES») PLAN IN ICA, PERU}

\begin{abstract}
In order to know the level of satisfaction of users from outpatient clinics in four public hospitals in the Ica region after the implementation of the "Zero Queues" plan, 1,067 users were surveyed by using an instrument that inquired about general characteristics, service qualification, and overall satisfaction. A global satisfaction level "very satisfied/satisfied" of $72.5 \%$ was found in the Support Hospital of Nazca; $70.6 \%$ in Hospital Santa María del Socorro of Ica; $72.1 \%$ in the Regional Hospital of Ica, and $73.7 \%$ in Hospital San Juan de Dios of Pisco. It is concluded that the implementation of the "Zero Queues" plan improved overall satisfaction, courteous treatment, compliance with health care hours, and the clarity and information provided above the expected standard.
\end{abstract}

Keywords: Patient satisfaction; Quality of health care; Ambulatory care; Health surveys; Peru (Source: MeSH NLM).

\section{INTRODUCCIÓN}

La satisfacción del usuario externo, es un indicador de gestión en las Instituciones Prestadoras de Servicios de Salud (IPRESS) y es el resultado de la diferencia entre percepción y expectativa ${ }^{(1)}$, la cual está influenciada por una serie de factores, como el estilo de vida, experiencias previas, expectativas, entre otros ${ }^{(2)}$, se considera una medida de control del funcionamiento del sistema sanitario ${ }^{(3,4)}$.

El Perú cuenta con una Política Nacional de Calidad en Salud con visión al 2020, donde la sociedad goce de un sistema de salud más efectivo ${ }^{(5)}$; en ese contexto el plan «Cero Colas» (PCC) se implementó de manera inicial en el Hospital Cayetano Heredia, con excelentes resultados ${ }^{(6)}$. Por

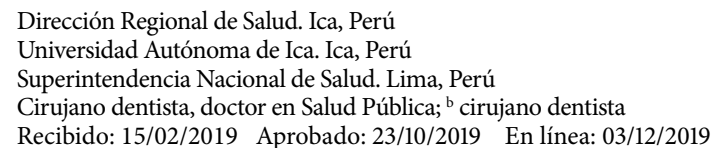

Citar como: Becerra-Canales B, Condori-Becerra A. Satisfacción de usuarios en hospitales públicos: experiencia del plan «Cero Colas» en Ica, Perú. Rev Peru Med Exp Salud Publica. 2019;36(4):658-63. doi: 10.17843/rpmesp.2019.364.4299. 
tal razón, el Ministerio de Salud (MINSA), decidió que esta experiencia debería replicarse en otros nosocomios a nivel nacional ${ }^{(7)}$. En efecto, a través de la Resolución Ministerial N811-2018/MINSA, se aprobó la directiva administrativa de implementación del PCC en las IPRESS del país; el cual consiste en fortalecer la infraestructura, equipamiento, materiales, recursos tecnológicos y humanos, soluciones informáticas, entre otras acciones, a fin de mejorar la calidad de la atención, disminuir los tiempos de espera e incrementar la satisfacción de usuario ${ }^{(8)}$.

Sin embargo, en un informe de La Contraloría General de la República de julio del $2018^{\left({ }^{(9)}\right.}$, se señala que el $72 \%$ de hospitales del MINSA no implementaron el PCC. Asimismo, en una medición basal realizada en marzo del 2017 en hospitales públicos de Ica que no implementaron el PCC se reportó, en promedio, un $63,1 \%$ de satisfacción y en otra evaluación en mayo del 2018 , en seis hospitales de la región se halló, en promedio, un $71,8 \%$ de satisfacción ${ }^{(10)}$.

Por lo tanto, la medición de la satisfacción posterior a la implementación del PCC en cuatro hospitales de la región Ica generará nueva evidencia. En ese contexto, conocer el grado de satisfacción del paciente, constituye un elemento necesario para garantizar la calidad y debe ser un indicador reportado por las IPRESS ${ }^{(11)}$. Los resultados obtenidos de su medición deben generar programas o proyectos de mejora continua de la calidad en salud, que incorporen acciones de monitoreo y seguimiento; asimismo, investigaciones como aportes al conocimiento.

El objetivo del estudio fue conocer el nivel de satisfacción de los usuarios de consulta externa de cuatro hospitales públicos de la región lca, en el marco de la implementación del PCC y determinar su variación respecto a una medición basal realizada en 2017 .

\section{EL ESTUDIO}

\section{DISEÑO DE ESTUDIO, POBLACIÓN Y MUESTRA}

Se realizó un estudio transversal en usuarios de consultorios externos del Hospital Apoyo de Nasca (HAN), Hospital Santa María del Socorro de Ica (HSMSI), Hospital Regional de Ica (HRI) y Hospital San Juan de Dios de Pisco (HSJDP). La población total se compone de 123035 usuarios atendidos entre junio y septiembre del 2018 (HAN=25 532, HSMSI =25 192, HRI=42 090 y HSJDP=30 221); el tamaño de la muestra fue calculado mediante el algoritmo matemático para poblaciones finitas, con un nivel de confianza del $95 \%$, precisión de $5 \%$ y una proporción esperada de $85 \%$. La muestra global fue de 1067 usuarios externos $(\mathrm{HAN}=222, \mathrm{HSMSI}=218, \mathrm{HRI}=365$

\section{MENSAJES CLAVE}

Motivación para realizar el estudio. La satisfacción del usuario externo, es un indicador de calidad de un sistema de salud efectivo, conocer su variación luego de la implementación del plan «Cero Colas» constituye una herramienta de gestión y una necesidad académica que debe ser cubierta con investigación.

Principales hallazgos. Los resultados preliminares, indican que la satisfacción de los usuarios de los hospitales públicos está mejorando, superando el estándar esperado.

Implicancias. Mejorar la calidad de la atención en salud es política sectorial, de ahí, que toda estrategia que contribuya a incrementar los niveles de satisfacción debe ser implementada y/o fortalecida en forma real y sostenida.

y HSJDP=262), seleccionadas mediante muestreo no probabilístico por conveniencia.

Se incluyeron usuarios de ambos sexos, mayores de 18 años, que podía ser pacientes, familiares o acompañantes y que brinden su aprobación para participar en la encuesta. Se excluyeron usuarios menores de 18 años, con algún tipo de discapacidad que les impida expresar su opinión, o que no deseen participar en la encuesta.

\section{INSTRUMENTO}

Se utilizó una escala modificada de la Encuesta Nacional de Satisfacción de Usuarios en Salud (ENSUSALUD) $2016^{(12)}$, el cual califica el servicio en base a diez preguntas con cinco opciones de respuesta (Muy bueno/bueno/ni bueno ni malo/malo/muy malo); además de una pregunta que mide la satisfacción global de usuario con cinco opciones de respuesta (muy insatisfecho/insatisfecho/ni satisfecho, ni insatisfecho/satisfecho/muy satisfecho). El instrumento fue sometido a validación por juicio de expertos, seleccionando a cinco coordinadores de gestión de la calidad, quienes evaluaron los ítems en términos de relevancia, coherencia, suficiencia y claridad.

\section{VARIABLES}

Se incluyeron variables generales (edad, sexo, consultorio externo y sede hospitalaria); variables que calificaron el servicio: el tiempo trascurrido desde que solicitó la cita hasta la fecha de consulta, la información que brindan sobre el estado de salud, el cumplimiento del horario de la atención médica, la atención del personal administrativo, el trato por parte del personal asistencial no médico (enfermeros, técnicos de enfermería, químicos farmacéuticos, entre otros), el trato por parte del personal médico, el tiempo de espera para la atención, la duración de la atención médica, 
los trámites administrativos y la claridad con que explicaron el tratamiento y pautas. Los resultados se presentan agrupados en tres categorías: muy bueno/bueno; ni bueno ni malo y malo/muy malo. Además de una variable destinada a medir la satisfacción global, cuyos resultados se presentan agrupados en tres categorías: muy satisfecho/ satisfecho; ni satisfecho ni insatisfecho e insatisfecho/muy insatisfecho.

\section{PROCEDIMIENTOS}

Los datos fueron recolectados durante noviembre y diciembre del 2018. Los usuarios fueron captados al finalizar la atención (farmacia, laboratorio, puerta de salida u otro). Para garantizar la calidad del llenado de las fichas, doce encuestadoras debidamente capacitadas y procedentes de los hospitales evaluados (tres de cada uno) fueron asignadas a otro nosocomio mediante sorteo; ellas aplicaron el instrumento teniendo en cuenta ciertas recomendaciones (saludar y presentarse, explicar el objetivo de la encuesta, obtener la aprobación verbal, enfatizar que la encuesta es anónima).

\section{ANÁLISIS DE DATOS}

Se preparó un libro de códigos, en base al instrumento y a la información obtenida, la cual fue incorporada en una base de datos, el proceso de crítica contempló la revisión de la información y la corrección de errores de digitación, la base de datos fue luego verificada y los errores depurados. El análisis estadístico descriptivo incluyó medidas de frecuencia, porcentajes y promedios. Para evaluar diferencias en el nivel satisfacción según las principales variables se utilizó la prueba de chi cuadrado. Para el procesamiento de datos, se utilizó el paquete estadístico "Statistical Package for the Social Sciences» para Windows versión 21.0. Se consideró un valor de $p$ $<0,05$ como estadísticamente significativo.

\section{ASPECTOS ÉTICOS}

El estudio se enmarca como una evaluación de un programa de salud pública, se solicitó el permiso al nosocomio y se indicó a los usuarios que su participación era voluntaria y anónima, obteniéndose el consentimiento verbal.

\section{HALLAZGOS}

Participaron 1067 usuarios de consultorios externos, la distribución según sexo fue homogénea (50,6\% masculino y $49,4 \%$ femenino), el $29,9 \%$ acudieron al consultorio de medicina, el $26,0 \%$ acudieron a cirugía (26\%) y el $23,1 \%$ acudieron a ginecología y obstetricia; el promedio de edad de los usuarios participantes fue de 41 años (Tabla 1).
Tabla 1. Características generales de los usuarios externos de hospitales públicos de Ica, Perú, 2018

\begin{tabular}{lcc}
\hline Variable & $\mathbf{n}$ & $\%$ \\
\hline Sexo & & \\
$\quad$ Masculino & 540 & 50,6 \\
$\quad$ Femenino & 527 & 49,4 \\
Consultorio externo & & \\
$\quad$ Medicina & 319 & 29,9 \\
$\quad$ Cirugía & 277 & 26,0 \\
$\quad$ Ginecología y Obstetricia & 246 & 23,1 \\
Pediatría & 102 & 9,6 \\
$\quad$ Otros & 123 & 11,5 \\
Sede hospitalaria & & \\
$\quad$ HAN & 222 & 20,8 \\
HSMSI & 218 & 20,4 \\
HRI & 365 & 34,2 \\
HSJDP & 262 & 24,6 \\
Edad, Media (DE) & $41,9(17,1)$ & \\
\hline
\end{tabular}

HAN: Hospital de Apoyo de Nasca, HSMSI: Hospital Santa María del Socorro de Ica, HRI: Hospital Regional de Ica, HSJDP: Hospital San Juan de Dios de Pisco

En cuanto a la calificación del servicio; el trato del personal médico fue calificado como «muy bueno/bueno» $(78,1 \%)$ en una proporción mayor respecto al trato del personal no médico $(63,2 \%)$. El cumplimiento del horario de atención médica $(71,1 \%)$, la claridad con que explicaron el tratamiento y pautas $(77,4 \%)$; la información que brindan sobre el estado de salud $(75,4 \%)$ y la duración de la consulta médica $(75,2 \%)$ fueron calificados como «muy bueno/bueno», por encima del estándar esperado (70\%).

Una proporción alta de usuarios calificó el servicio como «ni bueno ni malo»; mayormente referido al tiempo de espera desde la obtención de la cita hasta la fecha de consulta $(31,2 \%)$, a la atención del personal administrativo $(33,4 \%)$, al tiempo de espera para la atención $(32,4 \%)$ y a los trámites administrativos (34,4\%). Existe una proporción relativamente baja $(<10,8 \%)$ de usuarios que calificaron el servicio como «malo/muy malo» (Tabla 2 ).

Respecto al nivel de satisfacción global, se reporta como «muy satisfecho/satisfecho» al $72,5 \%$ en el $\mathrm{HAN}$, al $70,6 \%$ en el HSMSI, al $72,1 \%$ en el HRI y al $73,1 \%$ en el HSJDP (Tabla 3). Al comparar el nivel de satisfacción según sede hospitalaria, consultorio externo y edad, se encontraron diferencias significativas ( $p<0,001$ ); sin embargo, no se encontraron diferencias según sexo $(p=0,691)$.

En comparación con la medición basal realizada en marzo del 2017, utilizando la misma metodología y en una muestra de 753 usuarios (HAN=161, HSMSI=210, $\mathrm{HRI}=220$, HSJDP=162), el HAN reportó $85,7 \%$ de satisfacción; el HSMSI reporto 65,1\%, el HRI reporto 58,2\% y el HSJDP reporto $66 \%$; observándose un incremento en 
Tabla 2. Distribución de las calificaciones de los servicios de hospitales públicos de Ica, Perú, 2018

\begin{tabular}{|c|c|c|c|c|c|c|}
\hline \multirow{2}{*}{ Ítems } & \multicolumn{2}{|c|}{ Muy bueno /bueno } & \multicolumn{2}{|c|}{ Ni bueno, ni malo } & \multicolumn{2}{|c|}{ Malo/muy malo } \\
\hline & $\mathbf{n}$ & $\%$ & $\mathbf{n}$ & $\%$ & $\mathbf{n}$ & $\%$ \\
\hline $\begin{array}{l}\text { Tiempo de espera, desde la obtención de la cita hasta la fecha de } \\
\text { consulta médica }\end{array}$ & 619 & 58,0 & 333 & 31,2 & 115 & 10,8 \\
\hline La información que brindan sobre el estado de salud & 804 & 75,4 & 228 & 21,4 & 35 & 3,3 \\
\hline El cumplimiento del horario de la atención médica & 759 & 71,1 & 232 & 21,7 & 76 & 7,1 \\
\hline La atención del personal administrativo & 625 & 58,6 & 356 & 33,4 & 86 & 8,1 \\
\hline El trato por parte del personal asistencial no médico & 686 & 64,3 & 320 & 30,0 & 61 & 5,7 \\
\hline El trato por parte del personal médico & 833 & 78,1 & 202 & 18,9 & 32 & 3,0 \\
\hline El tiempo de espera para la atención & 630 & 59,0 & 346 & 32,4 & 91 & 8,5 \\
\hline La duración de la consulta o atención médica & 802 & 75,2 & 235 & 22,0 & 30 & 2,8 \\
\hline Los trámites administrativos & 602 & 56,4 & 367 & 34,4 & 98 & 9,2 \\
\hline La claridad con que le explicaron el tratamiento y pautas & 826 & 77,4 & 203 & 19,0 & 38 & 3,6 \\
\hline
\end{tabular}

tres hospitales (Figura 1) que, en promedio, representa el $9 \%$ respecto a la medición basal.

\section{DISCUSIÓN}

Los resultados preliminares de la implementación del PCC; indican que el nivel de satisfacción global de los usuarios es, en promedio $72,2 \%$, con un incremento respecto a la medición basal. El nivel más bajo se encontró en el HSMSI y el más alto en el HSJDP. Se destaca el incremento en el HRI; contrariamente a lo encontrado el HAN en donde la satisfacción está disminuyendo, debido presuntamente a la escasa sostenibilidad de las mejoras alcanzadas, la rotación continua de directores ejecutivos y al debilitamiento de estructuras de apoyo al Sistema de Gestión de la Calidad en Salud (SGCS). La distribución de los niveles de satisfacción global es heterogénea en los hospitales estudiados.

En contraste con los resultados de la ENSUSALUD 2016, que reporta un $66,7 \%$ de satisfacción en hospitales del MINSA (12) y considerando los resultados basales, se observa una propensión al incremento de la satisfacción, debido presuntamente a la mejora de la infraestructura, equipamiento, materiales, recursos tecnológicos y humanos, soluciones informáticas, entre otras acciones contempladas en la Directiva Administrativa N²51-MINSA/2018/ DGOS ${ }^{(13)}$. Estas medidas fueron implementadas, incluso antes de la aprobación de la directiva, tal como ocurrió en el
HAN, situación que justificaría el nivel de satisfacción más alto en la medición basal y cuya experiencia fue replicada por el HSJDP, HSMSI y HRI. EI SisGalenplus es un sistema informático que reduce el tiempo de espera, y ha sido implementado en cuatro de los cinco hospitales de la región con resultados alentadores ${ }^{(14)}$.

En efecto, los niveles de satisfacción en hospitales públicos de Ica, son superiores a los reportados por otros autores ${ }^{(15-18)}$ y considerado alto ${ }^{(19)}$. Sin embargo, el tiempo de espera desde la obtención de la cita hasta la fecha de consulta, el trato del personal asistencial no médico, el tiempo de espera para la atención y los trámites administrativos, aún mantienen menores niveles de calificación favorable; de ahí, que Gutierrez et al. señalan que el tiempo de espera condiciona un bajo nivel de satisfacción ${ }^{(20)}$. En contraste, la información que brindan sobre el estado de salud, el cumplimiento del horario de atención, el trato del personal médico, la duración de la consulta y la claridad con que explicaron el tratamiento y pautas; mantienen en promedio, una mayor calificación favorable; resultados que se corresponde con el estudio de Dámaso et al. quienes concluyen que el factor asociado al nivel de satisfacción fue, recibir y entender la explicación del médico ${ }^{(21)}$.

Finalmente, se evidencia que existe un incremento de la calificación del servicio como «muy bueno/bueno»; aunque existe un alto porcentaje de usuarios que lo califican como

Tabla 3. Nivel de satisfacción global de los usuarios externos de hospitales públicos de Ica, Perú, 2018

\begin{tabular}{|c|c|c|c|c|c|c|c|c|}
\hline \multirow{2}{*}{ Nivel de satisfacción } & \multicolumn{2}{|c|}{ HAN } & \multicolumn{2}{|c|}{ HSMSI } & \multicolumn{2}{|c|}{ HRI } & \multicolumn{2}{|c|}{ HSJDP } \\
\hline & $\mathbf{n}$ & $\%$ & $\mathbf{n}$ & $\%$ & $\mathbf{n}$ & $\%$ & $\mathbf{n}$ & $\%$ \\
\hline Muy satisfecho/ satisfecho & 161 & 72,5 & 154 & 70,7 & 263 & 72,1 & 193 & $\overline{73,6}$ \\
\hline Ni satisfecho, ni insatisfecho & 47 & 21,2 & 45 & 20,6 & 74 & 20,3 & 68 & 26,0 \\
\hline Insatisfecho/muy insatisfecho & 14 & 6,3 & 19 & 8,7 & 28 & 7,6 & 1 & 0,4 \\
\hline Total & 222 & 100 & 218 & 100,0 & 365 & 100,0 & 262 & 100,0 \\
\hline
\end{tabular}

HAN: Hospital de Apoyo de Nasca, HSMSI: Hospital Santa María del Socorro de Ica, HRI: Hospital Regional de Ica, HSJDP: Hospital San Juan de Dios de Pisco 


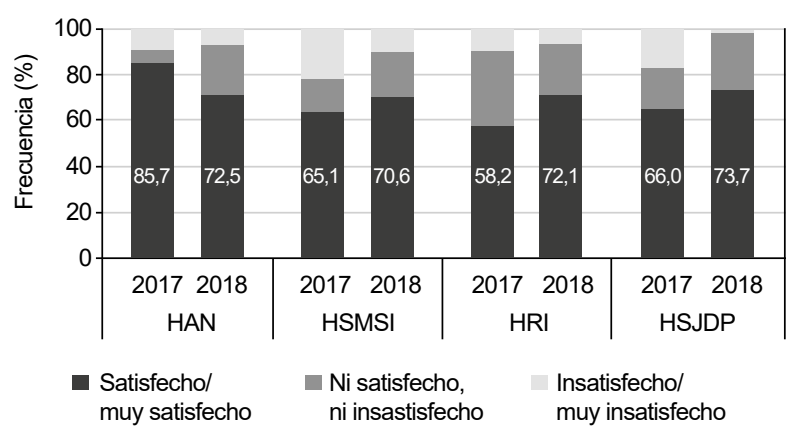

HAN: Hospital de Apoyo de Nasca, HSMSI: Hospital Santa María del Socorro de Ica, HRI: Hospital Regional de Ica, HSJDP: Hospital San Juan de Dios de Pisco

Figura 1. Niveles de satisfacción global de los usuarios externos de hospitales públicos de Ica, 2017-2018, Perú

«ni bueno ni malo» y un bajo porcentaje que lo califican como «malo/muy malo», situación reflejada también en la satisfacción global.

El estudio tiene ciertas limitaciones, se utilizó un instrumento de medición documental que mide una variable subjetiva (satisfacción del usuario luego de la implementación del PCC); sin embargo, es muy importante como medida de calidad de atención. Por otro lado, la percepción del usuario continuador podría estar sesgada por visitas anteriores, el tiempo que el usuario utiliza para desarrollar un cuestionario generalmente extenso de satisfacción, podría haber sido una limitación importante; sin embargo, esta fue superada con la selección de un instrumento relativamente corto.

En conclusión, la satisfacción de los usuarios de consultorios externos está mejorando progresivamente como resultado de la implementación del PCC; para lo cual, se requiere la participación del personal, el compromiso de la Dirección y el Equipo de Gestión en el otorgamiento de los recursos necesarios para su aplicación, garantizando la sostenibilidad de las acciones implementadas y el fortalecimiento del SGCS. Se recomienda implementar acciones y/o proyectos de mejora continua. Nuevas mediciones deberán ser realizadas para evaluar el impacto de las soluciones planteadas en el marco de la implementación del PCC, además de otros estudios que recorran la línea de investigación.

Material Suplementario: Disponible en la versión electrónica de la RPMESP.

\section{REFERENCIAS BIBLIOGRÁFICAS}

1. Mira JJ, Aranaz J. La satisfacción del paciente como una medida del resultado de la atención sanitaria. Med Clin (Barc). 2000;114 Suppl 3:26-33.

2. Car-Hill RA. The measurement of patient satisfaction. J Public Health Med. 1992;14(3):236-49.

3. Donabedian A. Evaluating the quality of medical care. Milbank Q. 2005;83(4):691-729. Doi: $10.1111 /$ j.1468-0009.2005.00397.x.

4. MonteagudoO,NavarroC,Alonso P,Casas R, Rodríguez L, Gracia J, et al. Aplicación hospitalaria del SERVQHOS: factores asociados a la satisfacción e insatisfacción. Rev Calid Asist. 2003;3(10):e2764. Doi: 10.1016/S1134-282X(03)77619-2.

5. Ministerio de Salud. Documento técnico: Política nacional de calidad en salud RM $\mathrm{N}^{\circ} 727-2009 / \mathrm{MINSA}$ [Internet]. Lima: Ministerio de Salud; 2009 [citado el 10 de enero del 2019]. Disponible en: http://bvs. minsa.gob.pe/local/minsa/1997.pdf

6. Menos tiempo en las colas [Internet]. El Peruano. 16 de julio de 2016 [citado el 9 de febrero de 2019]. Disponible en: https:// elperuano.pe/noticia-menos-tiempo-lascolas-41753.aspx
7. Consideran exitoso programa Cero Colas en hospital Cayetano Heredia [Internet]. Andina. 14 de mayo de 2016 [citado el 9 de febrero de 2019]. Disponible en: https:// andina.pe/agencia/noticia-consideranexitoso-programa-cero-colas-hospitalcayetano-heredia-612566.aspx

8. Ministerio de Salud del Perú. Minsa aprueba Plan Cero Colas en los establecimientos de salud a nivel nacional [Internet]. Lima: MINSA; 2018 [citado el 2 de febrero de 2019]. Disponible en: https:// www.gob.pe/institucion/minsa/noticias/18706-minsa-aprueba-plan-cero-colas-en-los-establecimientos-de-salud-a-nivel-nacional

9. La Contraloría General de la República. Operativo de control «Por una salud de calidad». Del 28 de mayo al 1 de junio de 2018 [Internet]. Lima: La Contraloría General de la República; 2018 [citado el 12 de febrero de 2019]. Disponible en: http://lfweb.contraloria.gob.pe/ BuscadorInformes/0/edoc/4811293/ Resum_Ejec_634-2018-CG-SALUDOCS.pdf

10. Becerra-Canales B. Satisfacción de usuarios en consulta externa de hospitales públicos de la región Ica, 2018. Rev Méd Panacea.
2018;7(3):89-92. Doi: https://doi. org/10.35563/rmp.v7i3.18.

11. Shimabuku R, Huicho L, Fernández D, Nakachi G, Maldonado R, Barrientos A. Niveles de insatisfacción del usuario externo en el Instituto Nacional de Salud del Niño de Lima, Perú. Rev Peru Med Exp Salud Publica. 2012;29(4):483-9.

12. Instituto Nacional de Estadística e Informática. Encuesta Nacional de Satisfacción de Usuarios en Salud 2016 [Internet]. Lima: INEI; 2016 [citado el 12 de febrero de 2019]. Disponible en: http://portal.susalud.gob.pe/wpcontent/uploads/archivo/encuestasat-nac/2016/INFORME_FINAL_ ENSUSALUD_2016.pdf

13. Ministerio de Salud del Perú. Directiva Administrativa No 251-MINSA/2018/ DGOS. Directiva para la elaboración e implementación del Plan Cero Colas en las Instituciones Prestadoras de Servicios de Salud Publicas. Resolución Ministerial No 811-2018/MINSA [Internet]. Lima: MINSA; 2018 [citado el 4 de febrero del 2019]. Disponible en: https://cdn.www. gob.pe/uploads/document/file/197306/ Resolucion-Ministerial-811-2018MINSA.PDF 
14. Gobierno Regional de Ica. «Lo logramos» Gestión 2015-2018 [Internet]. Ica: GORE-Ica; 2018 [citado el 4 de febrero del 2019]. Disponible en: http://www. regionica.gob.pe/web/images/stories2/ CADE/revista.pdf

15. Sihuin-Tapia EY, Gómez-Quispe OE, Ibáñez-Quispe V. Satisfacción de usuarios hospitalizados en un hospital de Apurímac, Perú. Rev Peru Med Exp Salud Publica. 2015;32(2):299-302.

16. Gerónimo-Carrillo R, Guzmán-Cejas L, Magaña-Olán L, Ramos-Rendón K. Calidad de servicio en la consulta externa de un centro de salud urbano de Tabasco. Salud Quintana Roo. 2016;9(35):11-5.

17. Godínez F, Carreto R, Ariza F, Maruris M, Cuevas A, Cabañas E. Nivel de satisfacción de usuarios de consulta externa en un hospital público. Psicol Salud. 2014;24(1):97-107.

18. García R, Gálvez N. Calidad de atención asociada a la satisfacción del usuario externo en los establecimientos asistenciales de salud: Minsa-Essalud, Chongoyape-Perú. Rev Tzhoecoen. 2016;8(2):1-10.

19. Infantes F. Calidad de atención y grado de satisfacción de los usuarios de consulta externa del Centro de Atención de Medicina Complementaria del Hospital III Iquitos-2016. Rev Peru Med integrativa. 2017;2(2):133-9. Doi: 10.26722/ rpmi.2017.22.55.

20. Gutiérrez E, Ramos W, Uribe M, OrtegaLoayza A, Torres C, Montesinos D, et al. Tiempo de espera y su relación con la satisfacción de los usuarios en la farmacia central de un Hospital General de Lima. Rev Peru Med Exp Salud Publica. 2009; 26(1):61-5.

21. Damasco B, Tucto J. Factores relacionados al nivel de satisfacción de los usuarios en la consulta externa en el Hospital II Red Asistencial Huánuco ESSALUD. Investig Valdizana. 2017;12(2):65-74. Doi: 10.33554/riv.12.2.141.

Correspondencia: Bladimir Becerra Canales Dirección: CC. HH La Angostura III Etapa H-10, Subtanjalla. Ica, Perú Teléfono: (+51) 956690060

Correo electrónico: icapredica@gmail.com

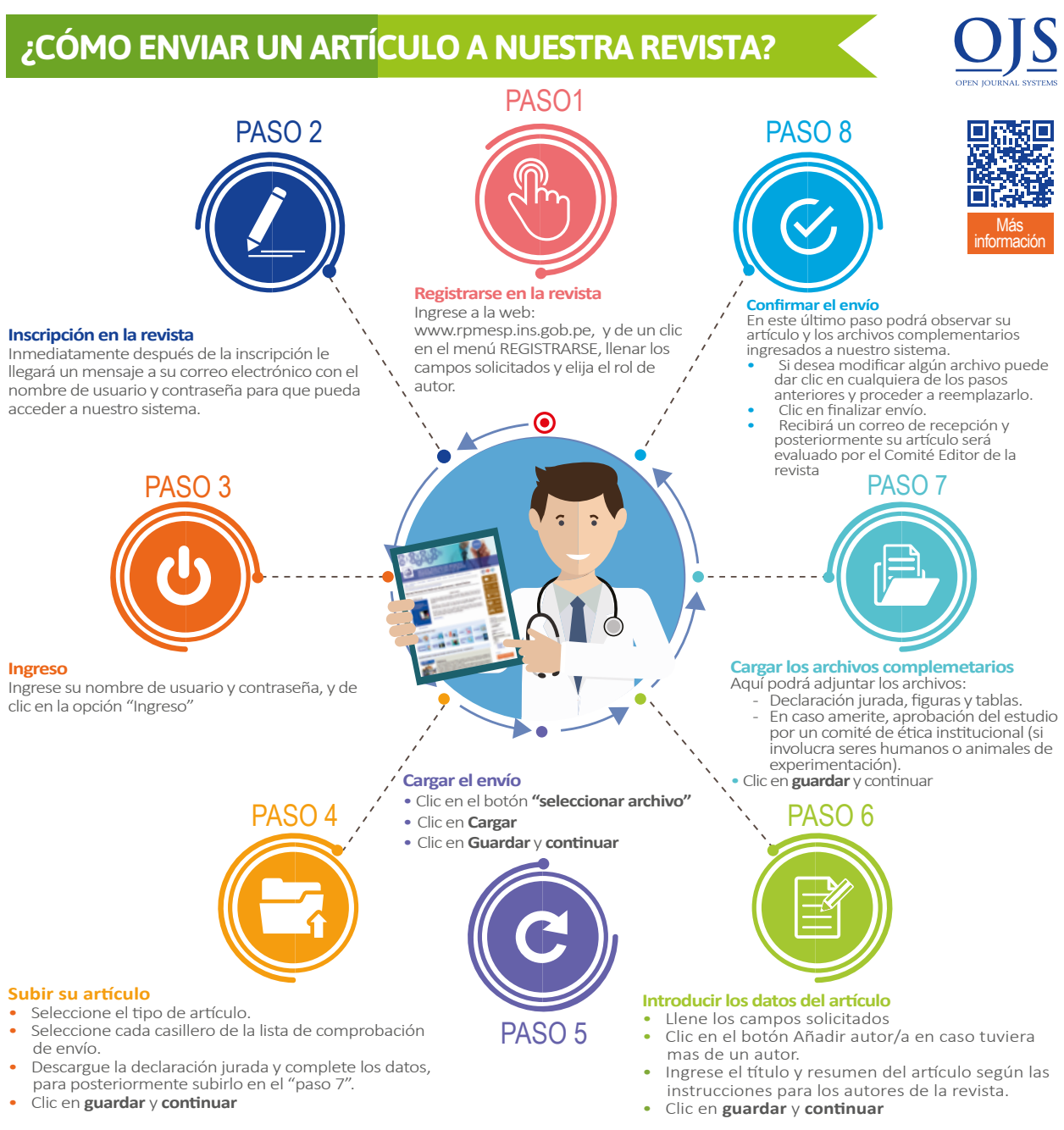

